\title{
ILC UNDULATOR BASED POSITRON SOURCE, TESTS AND SIMULATIONS
}

\begin{abstract}
Alexander A. Mikhailichenko, Cornell University, LEPP, Ithaca, New York
Abstract. An undulator based positron source allows generation of polarized positrons in quantities required by ILC. Here we describe the results of modeling and testing of elements for such a system.
\end{abstract}

Classification 3: Linear Colliders, Lepton Accelerators and New Acceleration Techniques, A03. Work supported by NSF grant PHY-0202078

\section{INTRODUCTION}

The scheme for polarized positron production was proposed a long time ago in a framework of VLEPP project [1]. The basis of the method is a two stage process, where at first stage the circularly polarized photons generated in helical electromagnetic field and then, at second stage, these photons converted into positrons and/or electrons in a thin ( half radiation length) target. Secondary particles carry longitudinal polarization transferred from the primary photon beam in accordance with theirs energy. In this first publication [1] the gammas considered to be generated by energetic particle in the following substances: in a field of electromagnetic wave, in static magnetic helical field of undulator and in crystals with helical dislocations (helical crystals). In [2] the laser radiation was considered as a specific example of an electromagnetic wave. With application of selection of energetic positrons only, the final polarization increased and defined by the length of undulator (as one needs to compensate partial collection of secondary positrons). For typical length of undulator $175 \mathrm{~m}$, the degree of polarization reaches $60 \%$ and it could reach $85 \%$ with $300 \mathrm{~m}$ long undulator. One peculiarity associated with helical undulator scheme is that this system is able to generate polarized positrons with degree of polarization $\sim 30 \%$ if no energy selection mechanism applied to the positrons at all.

The undulator scheme of positron production has been chosen as a baseline for ILC [3] accommodated from TESLA design [4]. One peculiarity here is that the beam, like in original VLEPP scheme is going through the undulator on its way to IP. One positive moment of this is that the beam can be made having small transverse dimensions as the emittance is small. This allows small aperture in undulator and hence makes engineering problem less severe. From the other hand nonlinear field of undulator could disturb this tiny emittance and polarization of this primary beam while it is going to IP. Considerations show that this is not a problem here however as the beam trajectory remains line-type with accuracy $\sim 1 / \gamma$ so the nonlinearities cancel each other. So the ILC scheme looks as it is represented in Fig.1

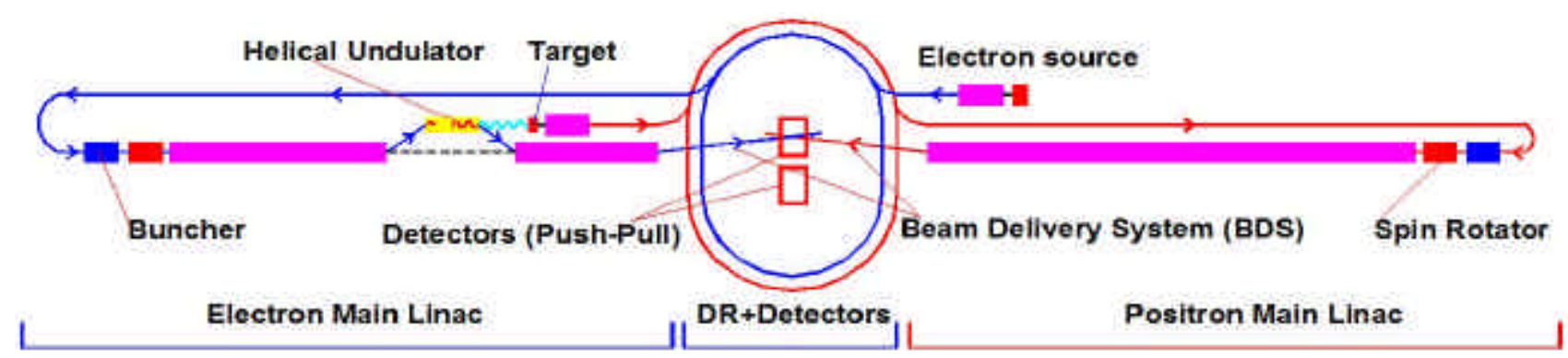

Figure 1: The basic scheme of ILC. 
Undulator located at the $150 \mathrm{GeV}$ mark in a chicane, as the energy of quanta radiated on harmonic number $n$ for the undulator with period $\lambda_{u}$ comes to $\sim 20 \mathrm{MeV}$ there

$$
E_{\gamma n} \cong \frac{n \cdot 2.48 \cdot\left(\gamma / 10^{5}\right)^{2}}{\lambda_{u}[\mathrm{~cm}]\left(1+K^{2}+\gamma^{2} \vartheta^{2}\right)}[\mathrm{MeV}]
$$

where the factor $K=e H \lambda_{u} / 2 \pi m c^{2} \cong 93.4 \cdot H[T] \cdot \lambda_{u}[m], \vartheta$ stands for the angle in direction to observer. Efficiency of conversion could reach such level, that two initial electrons generate in average three secondary positrons captured (1:1.5 conversion).

Minimal offset in chicane helps in reduction of radiated power (and power density, as the beam size is small) and makes possible emittance perturbation to be smaller also. This chicane could be arranged is the same tunnel without any additional extensions at all, se below.

So one can see that positron source is a complex system which includes a lot of different components and each of these components can be a subject of a separate talk.

\section{GENERATION OF POSITRONS}

The way to create the (circularly polarized) positron is shown in Fig.2.
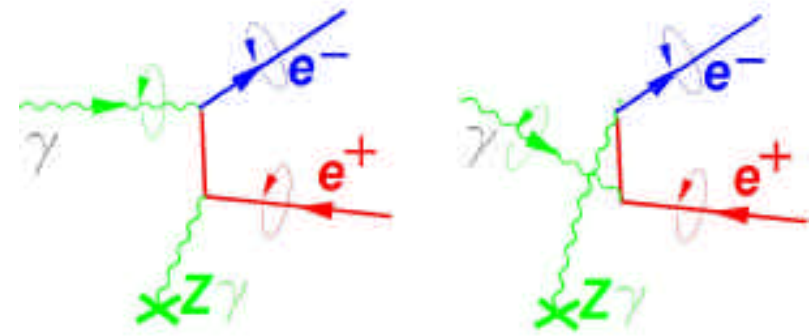

Figure 2: Diagrams describing the way to obtain a (polarized) positron.

As the only gamma-quanta can create an electron-positron pair, by all means the positron source must generate the gammas in necessary amounts, able to cover limited acceptance of collection optics. There are few possibilities on how to get gammas, Fig.3.
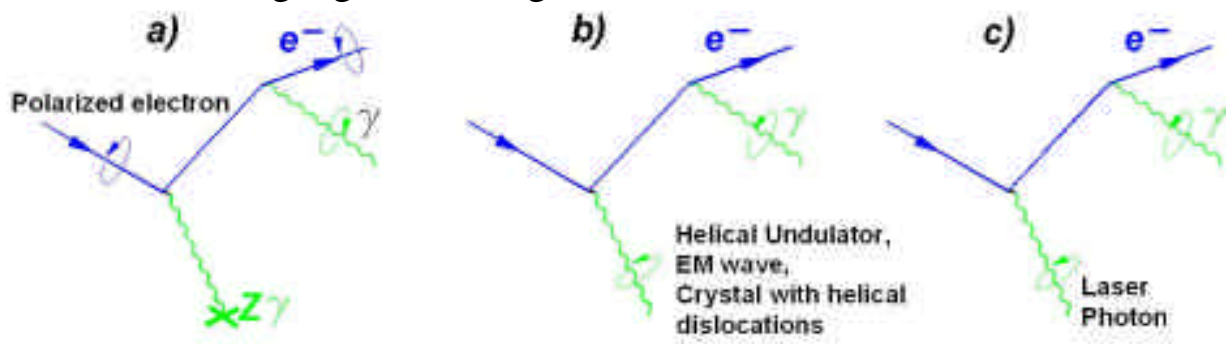

Figure 3: The way to obtain the (polarized) gamma-quanta. a)-the incoming electron is polarized [5], b)-the electron radiates in a helical electromagnetic field of broad nature [1], c)-the laser radiation appointed as a specific example of helical electromagnetic wave [2].

In ordinary conversion system the gammas created as a result of a cascade (shower), developed by the primary non polarized electron (Fig.3a with non polarized electron). This is so called generation of gammas by bremstrahlung. Shaking of electron here is going by the field of nuclei. This process is characterized by $X_{0}$-radiation length

$$
X_{0}^{-1} \cong 4 r_{0}^{2} \alpha \frac{N_{0}}{A} Z(Z+1) \ln \left(\frac{183}{Z^{1 / 3}}\right)\left[\mathrm{cm}^{2} / \text { gramm }\right],
$$


where $A$-is atomic weight of target substance, $N_{0} \cong 6.022 \cdot 10^{23}$ is the Avogadro number, $Z$ is the charge of nuclei, factor $Z(Z+1)$ takes into account atom electrons, $\alpha=e^{2} / \hbar c=1 / 137, r_{0}$ is a classic electron radius. Simplified picture of this process is represented in Fig. 4 below.

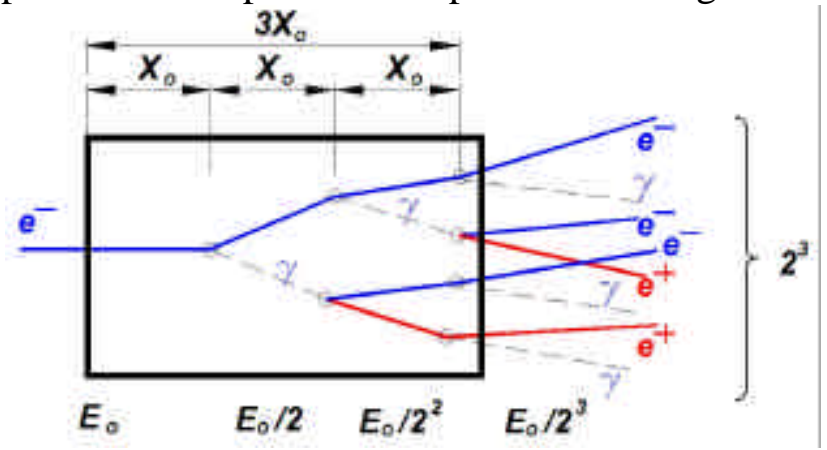

Figure 4: Cascade scheme for obtaining the gammas and positrons. Shown is the target with $3 \mathrm{X}_{\mathrm{o}}$.

After passing the distance $t$ (measured as a fraction of radiation length), the energy of the particles here becomes about $E(t) \cong E_{0} / 2^{\tau}$. The number of all particles, including photon is going to be $N_{t o t} \cong 2^{\tau}$, with number of positrons $N_{\text {pos }} \cong N_{t o t} / 3=2^{\tau} / 3$. The shower propagates inside target until the energy of particles reaches the critical one, $E_{\text {crit }} \cong 610 /(Z+1.24)$. So the shower reaches its maximum at the depth $\tau_{\max } \cong \ln \left(E_{0} / E_{c r i t}\right) / \ln 2$ with the number of the particles there about $N_{\max } \cong E_{0} / E_{c r i t}$. Transverse size of the cascade in maximum is of the order of Molière radius $R_{M} \cong X_{0} E_{s} / E_{c r i t}, E_{s}=\sqrt{4 \pi / \alpha} \cdot m c^{2} \cong 21.2 M e V-$ is a scale energy. Despite the thickness of target is significant in this type of conversion, the only outer layers are serving as the source of positrons, which energies in maximum are of the order of the critical, one, $\sim 10 \mathrm{MeV}$. The effective RMS depth $l$ of positron creation is

$$
l \cong<x x^{\prime}>/<x^{\prime 2}>
$$

where $x$ and $x^{\prime}$ stand for the transverse coordinate and its derivative, brackets mean average over all phase space. The last expression comes to $\sim 0.8 \mathrm{~mm}$ for $10-\mathrm{MeV}$ positrons. Knowing last number is important for description of target immersed in magnetic field, showing the principal depth, which magnetic field penetration tolerates the process.

In the scheme of positron production with gammas obtained by shaking primary electron (or positron) either in a field of static undulator, EM wave or in a laser field, Fig.3 b), c), the gammas represented by a separate source. So here the heating the target by primary electron component is absent and all target can be used for positron creation. Thus the target becomes having thickness of the order of latest layer in previous method.

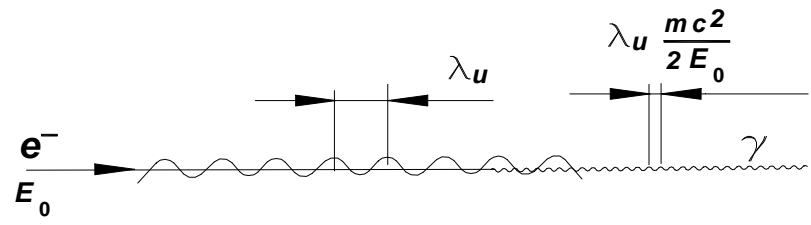

Figure 5: Generation of gammas by beamstrahlung by helical EM wave.

The number of the quants radiated by electron in the presence of the photons (real or virtual from wiggler) can be described in terms of effective length as the following

$$
N_{\gamma} \cong L \sigma_{\gamma} n_{\gamma}
$$


where $\sigma_{\gamma} \cong \frac{8 \pi}{3} r_{0}^{2}, n_{\gamma} \cong \frac{H^{2} \gamma^{2}}{8 \pi \hbar \omega}$ is the photon density in system of electron, $H$ is magnetic field value, $\omega=2 \pi c / \lambda$ is the frequency of the photon. So the length of interaction goes to $l_{\gamma} \cong 1 / \sigma_{\gamma} n_{\gamma}$. For the undulator having the length $L$

$$
N_{\gamma} \cong L r_{0}^{2} \frac{H^{2} \gamma^{2}}{\hbar \omega} \propto 4 \pi \alpha \frac{L}{\lambda_{u}} \frac{K^{2}}{1+K^{2}} .
$$

As the length of formation for undulator $\sim \lambda_{u}$ then this formula reflects the simple fact that the number of radiated photons is equal to the number of radiation lengths, $L / \lambda_{u}$. It is known, that particle radiates $\alpha$ photons on its passage through this distance.

So-called keep alive source required only if the positrons generated in independent loop (i.e. positrons generated in positron linac), otherwise the electron source linac can be appointed for the filling of positron ring using existing transfer lines with minimal additions allowing irradiation of positron target by electron beam passed through damping ring, extracted in transfer line connecting electron damping ring with the electron linac entrance. Further, near the target location, the electron beam is bending and focused onto the target with additional magnet system. This magnet system can be made compact; with high-field bending magnets as there are no obstacles from emittance dilution, while the beam irradiates the target, see Fig. 6 below.

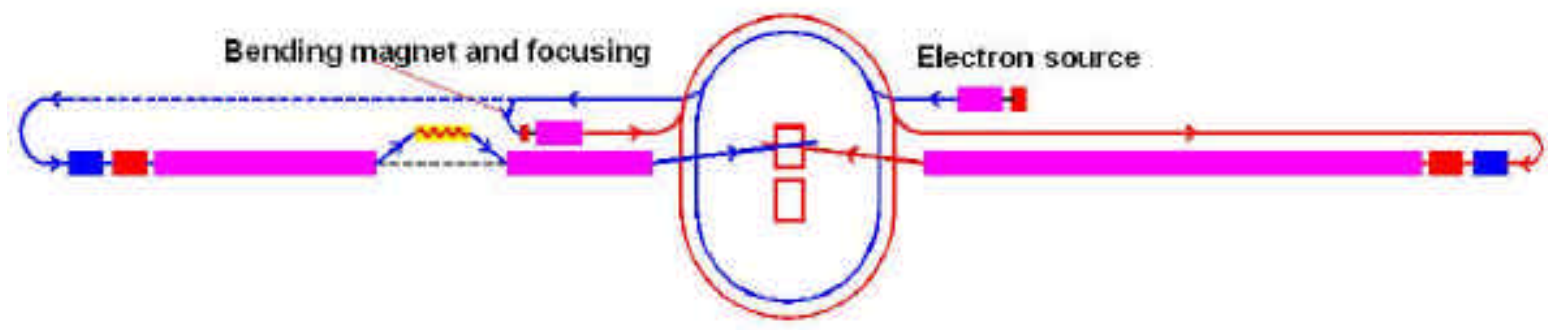

Figure 6: Possible operational scheme for filling positron ring by usage of electron source linac,

One additional comment can be made here. In [5] the method for polarized positron production was proposed implementing the usage of polarized electrons as a primary source (Fig. 3-a). During bremstrahlung, the longitudinally polarized electron radiates circularly polarized gamma at high edge of spectra. Further on, these polarized gammas become converted into electron-positron pairs in (the same) heavy target, similarly to conversion of undulator gamma-radiation. Polarized electrons obtained from the photocathode, in the same manner, as required for polarized electron source for ILC.

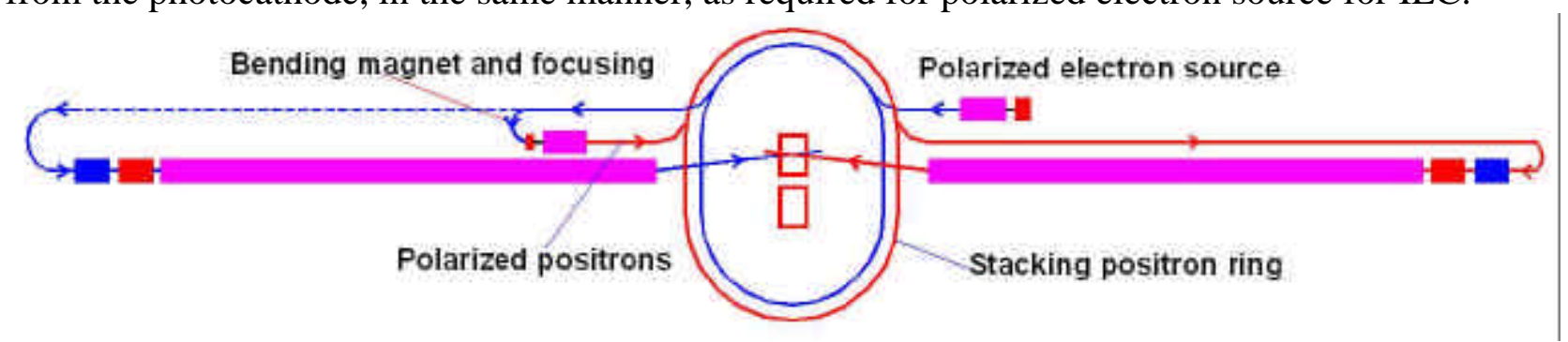

Figure 7: Usage of process from Figure 3, left for generation of polarized positrons. The stacking in positron ring required.

Efficiency of this method could reach $\sim 1.5 \%$ [5] i.e. each primary electron generates positron with probability 0.015 with polarization up to $\sim 80 \%$ of polarization of primary electron beam (which could be $\sim 9 \%$, so coming to $\sim 72 \%$ total). So to satisfy the requirements of ILC, the positron beam must be stacked in a damping (cooling) ring. But this is the same as for the mechanism for polarized gamma production by usage of Compton back scattering process (By other words the efficiency of processes 
shown in Figure 3 a) and c) are the same) [6]. For implementation of this method, one needs to use polarized electron source and insert a target in electron injection line; no lasers required at all, Fig.7. But still, the method with helical undulator is much more effective way to go. This comment can be considered as a serious argument against the Compton source of positrons for ILC.

\section{UNDULATOR CHICANE}

Parallel shift in chicane arranged with the help of two bending magnets at each side. Two radially focused quadrupoles at each side accomplish this bend. Total distance occupied by chicane comes to $\sim 350 \mathrm{~m}$ minimum; at this distance the RF structures more likely need to be removed. Minimal offset defined by the size of RF modules shadow further on of gamma-ray way as the target located at the distance $\geq 180 \mathrm{~m}$ from the end of undulator.

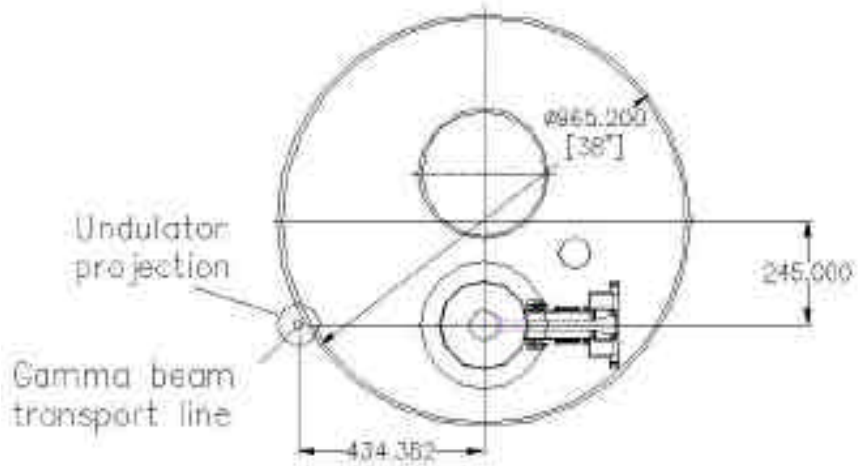

Figure 8: To the definition of minimal distance between chicane line and the main accelerator.

According to the procedure shown in Fig.8, minimal offset distance comes to 450mm. Bending magnets have active length $\sim 20 \mathrm{~m}$ each with bending radius $\sim 2 \mathrm{~km}$. Calculation of this chicane is rather challenging procedure as the tiny beam emittance makes SR radiation so severe, that without special measures this radiation can damage opposing wall of vacuum chamber [21]. This group of calculations includes also calculations of perturbation of emittance and polarization in undulator. The basic parameter here is the radius of helix, developed by electron in helical field of undulator $a \cong \lambda_{u} K / \gamma \sim 5 \cdot 10^{-7} \mathrm{~cm}$, which is $\sim 10^{-3}$ of the vertical beam size in undulator. As the nonlinearities are oscillating along the trajectory, they must be averaged over this transverse distance $\sim a$. Indeed, angular spread in radiation $\alpha \sim \sqrt{1+K^{2}} / \gamma$ is about 100 times bigger, than angular spread in a beam. This makes possible trimming of high harmonics by appropriate collimator, as all higher harmonics have zero intensity of radiation in forward direction.

\section{SIMULATION OF CONVERSION}

Diagram on Fig.2 needs to be considered with polarization of secondary positron (electron) as function of its energy $E_{+}$[7]. Analytically it can be expressed as [8]

$$
\vec{\zeta}=\xi_{2} \cdot\left[f\left(\frac{E_{+}}{E_{+\max }}\right) \cdot \vec{n}_{\mid}+g\left(\frac{E_{+}}{E_{+\max }}\right) \cdot \vec{n}_{\perp}\right]=\vec{\zeta}_{\mid}+\vec{\zeta}_{\perp}
$$

where functions $f$ and $g$ describe longitudinal and transverse polarization. This function behaves so that for higher energy of secondary particle it carries higher degree of polarization. What is important here is that maximal final degree of positron polarization defined by polarization of primary gammabeam as a factor. One can represent function $f$ in (6) as a Taylor series, taking into account, that $f(1)=1$ and $f^{\prime}(1)=0$ 


$$
|\zeta| \cong \xi_{2}\left(\frac{E_{+}}{E_{\gamma \max }}\right)+\xi_{2}^{\prime}\left(\frac{E_{+}}{E_{\gamma \max }}\right) \frac{E_{\gamma}-E_{+}}{E_{\gamma \max }}+\frac{1}{2 !}\left[\xi_{2}^{\prime \prime}+\xi_{2} \cdot f^{\prime \prime}(1)\left(\frac{E_{\gamma \max }}{E_{+}}\right)^{2}\right]\left(\frac{E_{\gamma}-E_{+}}{E_{\gamma \max }}\right)^{2}+\ldots
$$

One can see from here, that degree of polarization of particle having energy $E_{+}$is higher (for $E_{+}<E_{\gamma}$ ) or equal (for $E_{+} \cong E_{\gamma}$ ) to the degree of photon polarization having the same energy.

Main characteristics of Undulator Radiation (UR) are the energy of quants (1), spectral photon density $d N_{\gamma} / d E_{\gamma}$ and it polarization as this parameter appears as a factor in final polarization of positron (6). Expression for spectral density of radiation for undulator having length $L$ has a form [18] ,

$$
\frac{d N_{\gamma}}{d E_{\gamma}}=\sum_{n} \frac{d N_{\gamma n}}{d E_{\gamma}}=\frac{\alpha K^{2} L}{\hbar c 2 \gamma^{2}} \sum_{n=1}^{\infty} F_{n}(K, s),
$$

where $s=E_{\gamma n} / E_{\gamma n \max }, E_{\gamma \max }$ defined by (6) for $\vartheta=0$,

$$
F_{n}(K, s)=J_{n}^{\prime 2}(n \kappa)+\frac{1+K^{2}}{4 K^{2}} \frac{(2 s-1)^{2}}{s(1-s)} J_{n}^{2}(n \kappa),
$$

$\kappa=2 K \sqrt{s(1-s) /\left(1+K^{2}\right)}, J_{\mathrm{n}}$ stands for the Bessel function of the first kind. Differential cross section referred to the radiation length unit can be represented as the following $d \sigma\left(E_{\gamma}, E_{+}\right) \cong \sigma_{0} d E_{+} / d E_{\gamma}$, where [22]

$$
\sigma_{0} \cong A /\left(N_{0} X_{0}\right) G\left(E_{+}, E_{\gamma}\right)
$$

stands for total cross-section of photon absorption at the radiation length growing up to 7/9 at high energy,

$$
G(x)=x^{2}+(1-x)^{2}+\frac{2}{3} x(1-x)-x(1-x) /\left(9 \ln \left(183 Z^{-1 / 3}\right) \text {. Variation of } \sigma_{0}\right. \text { is equivalent of slow variation with }
$$

energy of the interaction length and requires appropriate correction of target thickness for better efficiency.

The number of positrons generated by a single photon in the target layer having thickness $d \tau$ at the depth $t$ counted from the entrance

so the spectral density becomes

$$
\frac{d^{2} N_{+}}{d E_{+} d \tau}=\frac{1}{\sigma_{0}} \iint \frac{d \sigma\left(E_{\gamma}, E_{+}\right)}{d E_{+}} \frac{d^{2} N_{\gamma}}{d E_{\gamma}} d E_{\gamma},
$$

$$
\frac{d N_{+}}{d E_{+} d \tau} \cong 0.4 \frac{\alpha K^{2} L}{\gamma^{2} \hbar c} \frac{7}{9}\left(1-E / E_{\gamma 1}\right)\left(1-e^{-7 \tau / 9}\right)
$$

For $E_{0}=150 \mathrm{GeV}, L=150 m, K^{2}=0.1, \tau \cong 0.5$ (rad units)

$$
\frac{1}{N_{t o t}} \frac{d N_{+}}{d E_{+}} \cong 0.2[1 / \mathrm{MeV}]
$$

More detailed analytical formula for efficiency of conversion per each initial electron taking into account finite length of undulator stands [18]

$$
\Delta N_{+1} \cong 2 \cdot 10^{-2} \chi^{2} M \delta \frac{K^{2}}{1+K^{2}} \frac{z_{f}}{z_{i}}\left(1-\zeta_{c a p}\right),
$$

where $\chi$ is a is a fraction of what is the target radius in respect to the size of the gamma spot at the target distance, $z_{i, f}$ are the coordinates of undulator end and beginning calculated from the target position, $1-\zeta_{c a p}$ is efficiency of geometric capture of positrons, $M=L / \lambda_{u}$ is total amount of undulator periods, $d$ is thickness of target in radiation length. For $\chi=1 / 2, M=10^{4}, \delta=0.2, K=1, \zeta_{\text {cap }}=0.7$, $z_{f}=M \lambda_{u}=2 z_{i}$, total amount of positrons per electron in undulator comes to $\Delta N_{+1} \cong 3$. 
Although analytical calculations found to be accurate, from the very beginning, numerical calculations were thought as a supplemental way to go.

One general question is how low K-factor could be? One can see from (5) that the number of photons is extremely sensitive to the $K$ factor value. From the other hand with increasing the $\mathrm{K}$ value the content of higher harmonics also increased. At $K^{\sim} 0.7$, the power radiated at the first harmonic comes to $50 \%$ of total one. Radiation at harmonics has proportionally higher photon energy, what makes collection of particles more difficult.

To answer these questions few numerical code were used such as KONN [21], CONVER [23], OBRA [26], [27], In particular KONN is start to end computer code realizing Monte-Carlo simulation of radiation in undulator, conversion in target, collection by Li lens and further acceleration.

Argonne Laboratory also began modeling of positron conversion with undulator using EGS4, Geant4 and Fluka [19]. Same type of calculations carried at SLAC [28]

Answer to this question obtained is that $\mathrm{K}<0.4, \lambda_{u}=1 \mathrm{~cm}, \mathrm{~L} \sim 175$ is enough for 1:1.5 conversion in positrons with $\sim 60 \%$ polarization for collection with Lithium lens (see below). Bigger K 0.9 allows having L 30m with polarization $\sim 40 \%$.

\section{TARGET}

Power dissipated in a target with traditional method by direct electron/positron conversion becomes so big, that it is not practical for ILC. That is why positron production scheme with undulator was chosen as a baseline for ILC. Even so the target problem remains serious.

The base line for now, is the Titanium rim-target having diameter $\sim 1 \mathrm{~m}$ spinning at $500 \mathrm{rpm}$ [20]. Thickness of this rim comes to $\sim 1.42 \mathrm{~cm}$, close to $\mathrm{X}_{0} / 2$ for Ti. Such a big thickness introduces additional difficulty for collection optics, which now needs to have the focal depth of the order of the thickness of target.

On possible solution of this could be a sandwich type target [21], Fig.9.

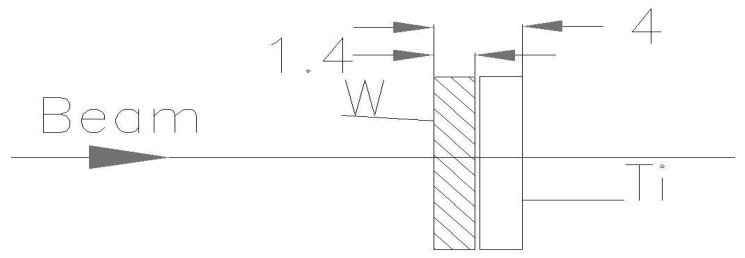

Figure 9: Two-layer target with $\mathrm{W}$ as the first one. Dimensions are given in $\mathrm{mm}$.

Other possibility is a liquid metal target, $\mathrm{Pb} / \mathrm{Bi}$ or $\mathrm{Hg}$ [. In this type of target the metal confined in a profiled duct having Be flange at the exit side of this duct.

Temperature and pressure dynamics in a target governed by equations

$$
\nabla(k \nabla T)+\dot{Q}=\rho c_{V} \dot{T}, \quad \ddot{P}-\nabla\left(c_{0}^{2} \nabla P\right)=\Gamma(V) \dot{Q} / V_{0},
$$

where $k$ stands for thermal conductivity, $\dot{Q}\left[\right.$ Watts $\left./ \mathrm{cm}^{3}\right]$-density of energy deposition, $c_{V}$ stands for the heat capacity $c_{0}$ is a speed of sound in a target material, $\Gamma(V)=V / c_{V}\left(\partial P / \partial T_{V}\right)$ characterizing the ratio of the thermal pressure to the specific thermal energy $\varepsilon_{T} / V$ called Grüneisen coefficient.. Calculations show that the average power deposition in a target $\sim 5 \mathrm{~kW}$. So every second $Q=5 \mathrm{~kJ}$ is deposited there. We created numerical model for solving (15) using FlexPDE with the moving source

$$
\dot{Q}=\sum_{i} \frac{2 c Q_{\text {bunch }}}{\pi \sqrt{\pi} \sigma_{z} \sigma_{\perp \gamma}^{2} l_{T}} \frac{z}{l_{T}} \exp \left(-\frac{\left(z+z_{0}-c\left(t-i \cdot t_{0}\right)\right)^{2}}{\sigma_{z}^{2}}\right) \cdot \exp \left(-\frac{r^{2}}{\sigma_{\perp \gamma}^{2}}\right)
$$


$Q_{\text {bunch }}$ stands for the energy deposited by single bunch, $i$-numerates the bunch, $z_{0}$ initial displacement. Expression normalized so that for the single bunch

$$
\int_{0}^{\infty} d t \int_{0}^{\text {Volume }} \dot{Q}(r, z, t) d V=Q_{\text {bunch }}
$$

Some results of this modeling show that temperature rise could be kept at the level of $125^{\circ} \mathrm{C}$.

By introduction of focusing and/or some steering of beam in undulator, one can artificially increase the gamma-spot size on the target. The energy $Q_{b u n c h}$ deposited by the bunch in the target is $Q \cong 0.15-0.2 J$ depending on details of focusing in undulator. So the pressure existing at the very first moments comes to $[11]$

$$
p_{T}=\Gamma(V) \frac{\varepsilon_{T}}{V} \cong \Gamma(V) \frac{Q}{\pi \sigma_{\perp \gamma}^{2} l_{T}} \cdot \frac{z}{l_{T}},
$$

where $z$ coordinate runs from the entrance of target. As the Grüneisen coefficient for typical case $\sim 2.4$ then the thermal pressure at the first moment comes to $\sim 1 \mathrm{kbar}$ level.

In numerical model the flanges supported at different temperatures (20 and $250{ }^{\circ} \mathrm{C}$ respectively) and the properties of Mercury were substituted here. This model, showing dynamics of heating indicate good agreement with analytical estimations.

Compton electrons practically do not input to the heating; indeed, positrons and electrons from pairs generated in equal quantities and, hence, heat the target equally.

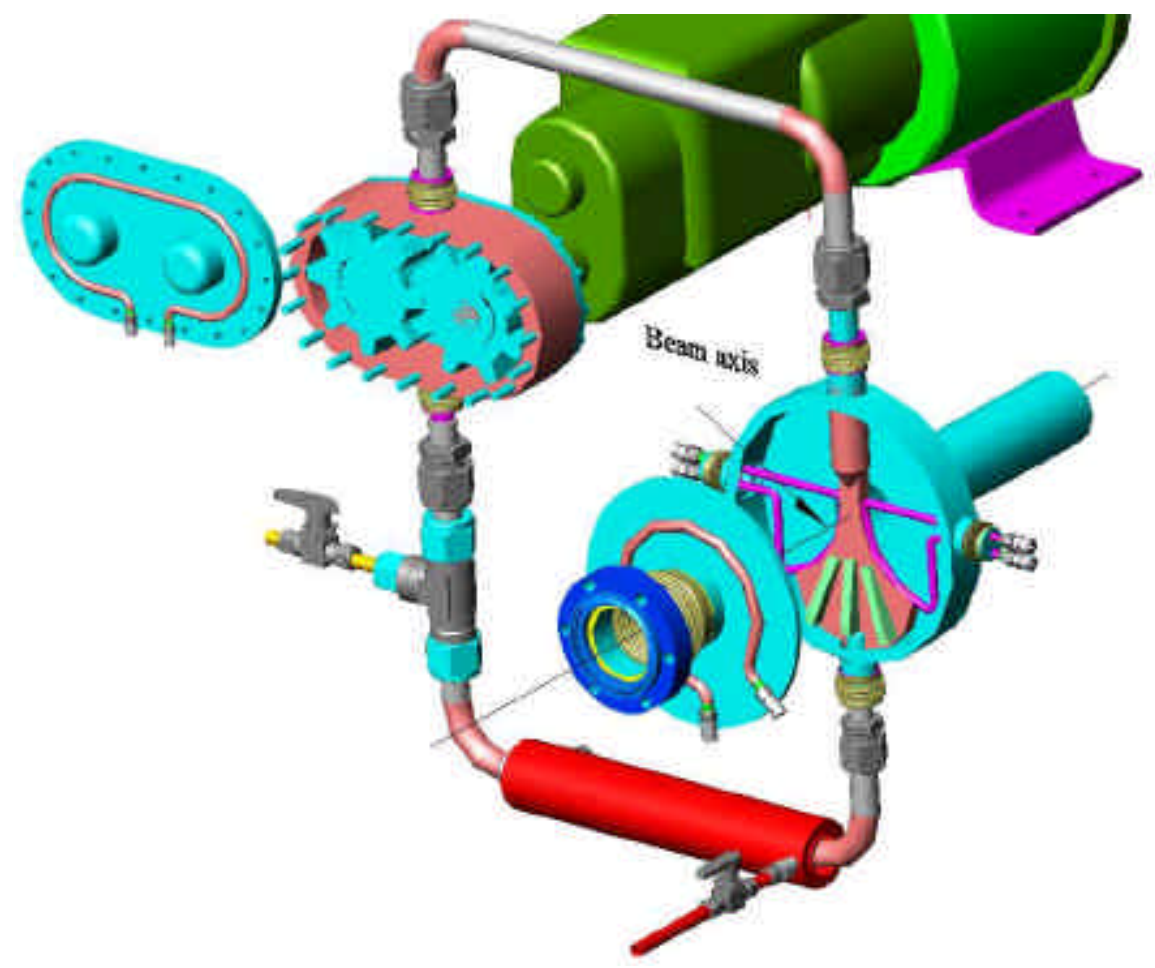

Figure 10: Liquid metal target system shown with jet chamber, pump and heat exchanger.

\section{COLLECTION OPTICS}

Usage of collection optics has a peculiarity here as the spinning target rim perturbs magnetic field as result of eddy currents in moving metal [25]. So collection optics must be field free in region of target. 


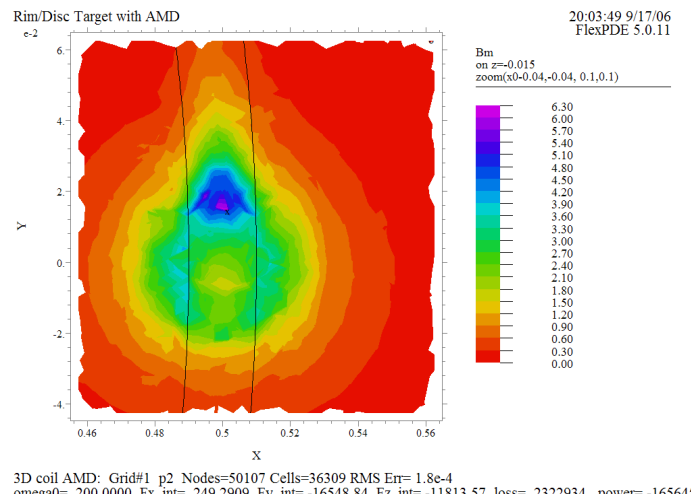

Figure 11: Top view to the rim-target with distribution of magnetic field modulus. Rim is spinning counterclockwise. Drag is seen clearly.
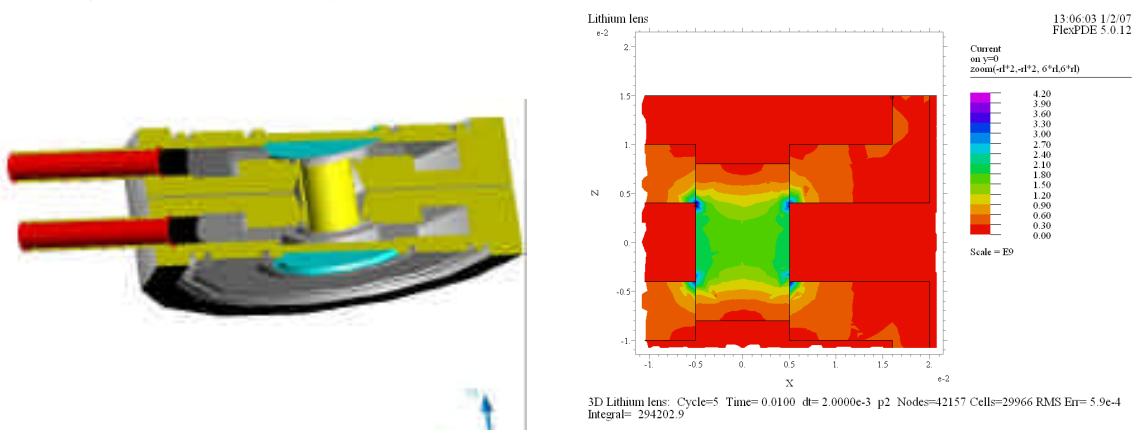

Figure 12: Lithium lens, left. Current distribution, right

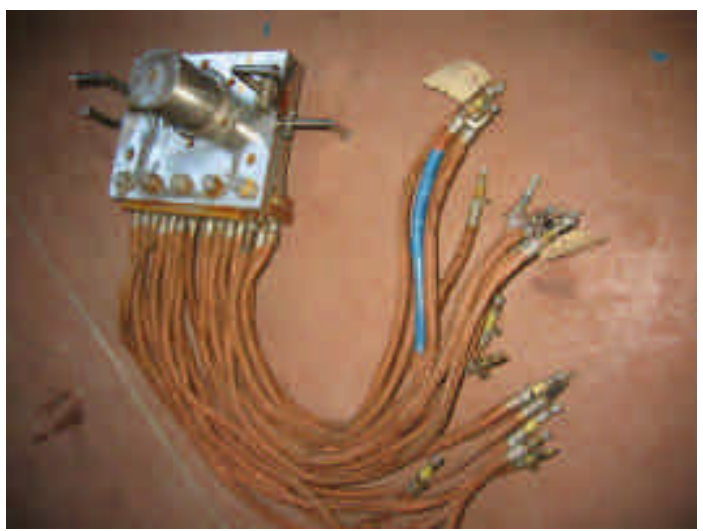

Figure 13: The Lithium lens in use at Novosibirsk BINP (Photo-Courtesy of Yu. Shatunov).

Solenoidal dual layer lens can be made with same focal distance as the Lithium lens, $f \sim 1 \mathrm{~cm}$ as the energy of secondary particles are $\sim 15 \mathrm{MeV} \pm 5 \mathrm{MeV}$.

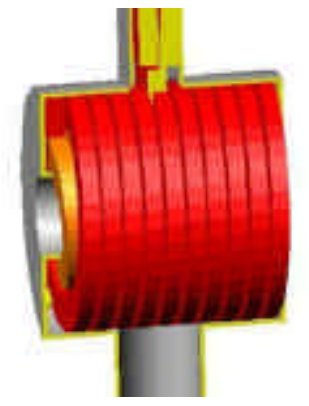

Figure 14: Dual layer lens cooled by oil. 
Accelerating structure is important component of positron conversion system. Structure immersed in solenoidal field having maximal value up to $40 \mathrm{kG}$, so the room temperature structure with big aperture must be used here. Such structures with appropriate parameters are under development [10], [20]. Structure developed in [20] has one input located close to the target side, indeed the RF power input in [10] made in a symmetric way by usage of two waveguides. What is important here is to locate RF input at the far end of the structure counted from the target.

\section{TEST OF UNDULATORS}

Undulators satisfying requirements of positron conversion system very fabricated and tested in Novosibirsk in 1986 as a part of VLEPP program [11]. Pulsed undulator tested had aperture 4mm, period $6 \mathrm{~mm}$ and could reach $K=0.35$ with feeding current $\sim 10 \mathrm{kA}$. SC undulator had period $10 \mathrm{~mm}$, aperture $\sim 6 \mathrm{~mm}$ and could reach $K \sim 0.6$. Basically these designs served as prototypes for design of undulator for E-166 experiment (see below) and for SC undulators developed at Cornell.

Few undulators were tested at Cornell. Basically they can be grouped in two categories as having $10 \mathrm{~mm}$ and $12 \mathrm{~mm}$ periods. Helical iron yoke of appropriate period used in all undulators designs so far. All undulators tested have clear aperture $8 \mathrm{~mm}$. Maximum $K$ factor reached for $10 \mathrm{~mm}$ period undulator is $K=0.467$ and $K=0.83$ for $12 \mathrm{~mm}$ period. According to our calculation any of these undulators can satisfy 1:1.5 efficiency of conversion. However we considering the reduction of aperture down to $6.25 \mathrm{~mm}$ which allows having $K=0.7$ for $10 \mathrm{~mm}$ period and $K=1.2$ for $12 \mathrm{~mm}$ period. This might be useful for initial period of tuning the ILC. And low $K$ factor helping in obtaining higher degree of polarization could be installed later on by lowering the feeding current.

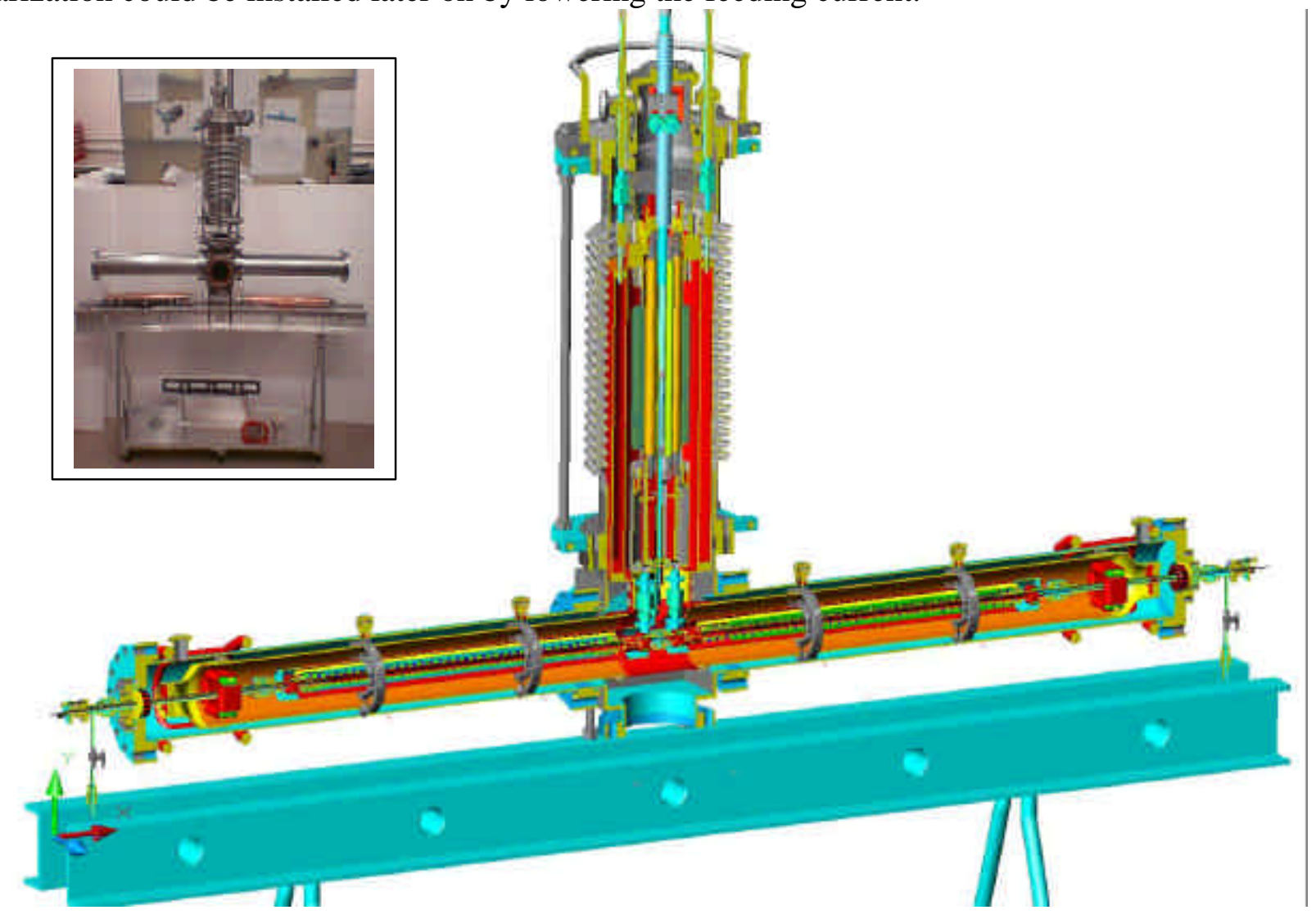

Figure 15: The cross-section of undulator module under assembling in Cornell LEPP. This $1.5 \mathrm{~m}$ long prototype model has all elements carried by full 4-m long prototype. 


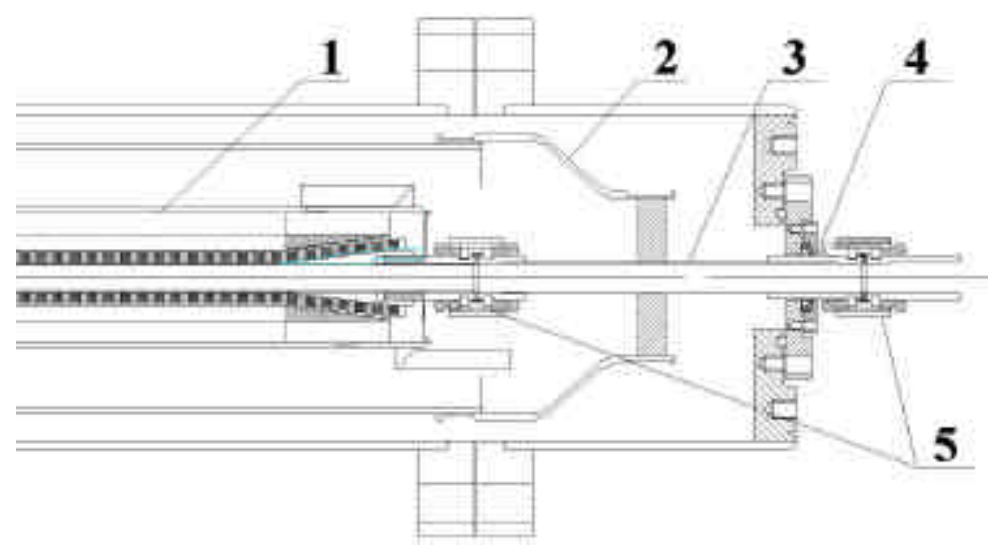

Figure 16: Schematics of transition region between cold mass and the room temperature flange in Cornell undulator. 1 - cold mass, $70{ }^{\circ} \mathrm{K}$ shield, 3-StSteel thin wall tube, 4-Wilson type sealant, 5Conflat $^{\circledR}$ joints.

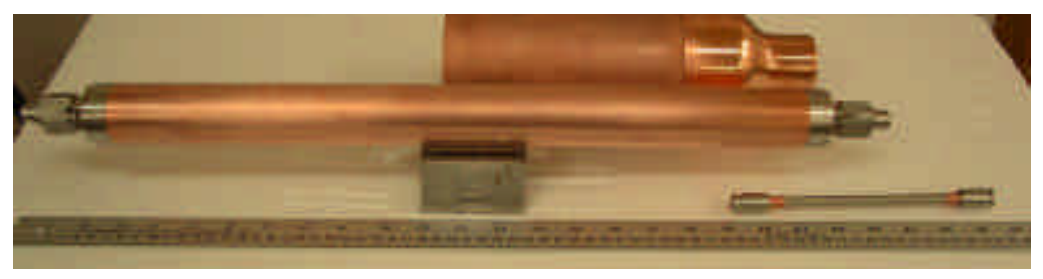

Figure 17: Cold mass assembled; elements of 70K shield and transition StSteel tube.

Much attention paid for smooth transition between modules. One can see from Fig.20, that the Copper vacuum chamber in region of cold mass with a help of metal gasket joint to the stainless steel tube covered by thin Copper layer inside. At some distance between LHe cold mass and room temperature flange, there is c thermal contact with $70{ }^{\circ} \mathrm{K}$ Copper shields. Diameters of copper tube in cold mass and the stainless steel transition one are the same so the perturbation due to wake fields is minimal. This transition required only between long segments as for mostly length, the cold mass of one section joint directly to another one with metallic gaskets.

Indeed, in design [9] the transition is rather long and has significant variations in diameter see Fig. 16.

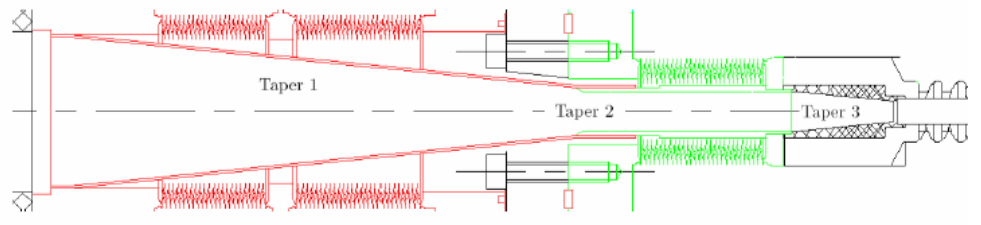

Figure 18: The transition in Daresbury model [9]. Cold mass is at the right.

More detailed description of development undulator here one can find in [29].

\section{TEST OF CONVERSION SYSTEM}

Recently the test of polarized positron production in experiment E-166 [17] was done successfully. Polarization of positrons $\sim 85 \%$ measured in good agreement with calculations. We expect detailed publication soon. Polarization measured by usage of spin dependence in cross section of circularly polarized gammas, propagating in magnetized Iron.

Targets were installed on remotely movable device having few slots for installation of targets of different materials and thickness. 
One important element of test was a test of target of different thickness and materials. Was found, that $\mathrm{W}$ target gives $45 \%$ higher yield than the Ti target of the same thickness $\left(0.5 \mathrm{X}_{0}\right)$.

\section{CONCLUSIONS}

Conversion system using undulator as a source of circularly polarized photons delivers polarized positrons at the exit in the amounts $>1.5$ per each initial electron/positron at the entrance. This efficiency can be obtained with relatively low $\mathrm{K}$ factor $<0.4$. Main beam of electrons after reaching 150 $\mathrm{GeV}$ directed in undulator installed in chicane line with minimal deflection from the linac axis. Aperture of undulator due to small dimensions of the driving (primary) beam could be 6-8mm only. Period of undulator might be 10-12 $\mathrm{mm}$ and this well supported by models developed and tested at Cornell and at Daresbury.

Polarization in both beams is extremely powerful tool for High energy physics as it delivers a possibility to prepare more cleanly initial condition and suppress mostly backgrounds. We are concluding that if Linear Collider will be built in some time at all, it must be able to collide both positrons and electrons polarized. Undulator needs to be made with sections $\sim 4 m$ each with total length $\sim 200 \mathrm{~m}$. Longer undulator-higher polarization can be achieved. With $300 \mathrm{~m}$-long undulator polarization can reach $80 \%$. Polarized electrons could be obtained by the same way also.

Experiment E-166, which completed at SLAC, eliminated any doubts about polarized positron production possibilities.

\section{REFERENCES}

[1] V.E.Balakin, A.A.Mikhailichenko, "Conversion System for Obtaining Highly Polarized Positrons and Electrons at High Energy", Budker INP 79-85, Sept. 13, 1979.

[2] E.G. Bessonov, "Some Aspects of the Theory and Technology of the Conversion Systems of Linear Colliders", $15^{\text {th }}$ International Conference on High Energy Accelerators, Hamburg, 1992, p.138.

[3] For ILC Reference Design Report we address to: http://www.linearcollider.org

[4] TESLA: "Conceptual Design of a $500 \mathrm{GeV} e^{+} e^{-}$Linear Collider with Integrated X-ray Laser Facility”, DESY 1997-048, ECFA 1997-182, Vols.1-2, Ed.: R.Brinkmann, G.Materlik, J.Rossbach, A.Wagner, May 1997.

[5] E.A.Bessonov, A.A.Mikhailichenko,"A Method of Polarized Positron Production", THP071L, Published in EPAC96, Barcelona, June 9-14, 1996. Proceedings. p.1516, see: http://accelconf.web.cern.ch/AccelConf/e96/PAPERS/THPL/THP071L.PDF.

[6] T.Omori," ERL based Compton $e^{+}$source for ILC", ILCWS2007, Proceedings, http://lcws07.desy.de/

[7] H.Olsen, L.Maximon, Phys. Rev., 1959, 114, 887.

[8] V.N.Baier, V.M.Katkov, V.S.Fadin, "Radiation of relativistic electrons", Moscow, Atomizdat, 1973.

[9] A. Birch," ILC Positron Source Helical Undulator Update", a Talk at ILCWS2007, Hamburg, see ILCWS website- http://lcws07.desy.de/.

[10] L.V. Kravchuk, V.A. Moiseev, A,N. Naboka, V.V.Paramonov, A.K.Skasyrskaya, "INR Activity in the Accelerating Cavities and Studies", a Talk at ILCWS2007, Hamburg, see ILCWS websitehttp://lcws07.desy.de/.

[11] A. Mikhailichenko, "Conversion System for Obtaining Polarized Electrons and Positrons at High Energy”, Dissretation, Novosibirsk 1986, Translation in CBN 02/13, Cornell, 2002.

[12] E.A. Perevedentsev, V.I. Ptitsin, Yu.M. Shatunov, "Spin behavior in Helical Undulator". Int. J. Mod. Phys. A, Proc. Suppl. 2A (1993) 170-172.

[13] E.A. Kushnirenko, A. A. Likhoded, M.V. Shevlyagin, "Depolarization Effects for Collisions of Polarized $e^{+} e^{-}$beams", IHEP 93-131, SW 9430, Protvino 1993.

[14] A. Mikhailichenko, CBN 02-10, Cornell LEPP,2002. 
[15] Yu. Ivanyushenkov, et.al, "Development of Helical SC Undulator for a Polarized Positron Source", PAC2005, Knoxville, Proceedings, pp.2295-2297.

[16] A. Mikhailichenko, "Positron Source for ILC. A Perspective", CBN 06-1, Cornell 2006, http://www.lns.cornell.edu/public/CBN/2006/CBN06-1/CBN06-1.pdf

[17] G. Alexander et al.,"Undulator-based production of polarized positrons: A Proposal for the 50GeV beam in the FFTB", SLAC-TN-04-018, SLAC-PROPOSAL-E-166, Jun 2003. 67pp.

[18] E.A.Bessonov, A.A.Mikhailichenko, "Some Aspects of Undulator Radiation Forming for Conversion System of the Llinear Collider”, Budker INP-1992-43, Jun 1992. 26pp; see http://ccdb4fs.kek.jp/cgi-bin/img_index?9302032

[19] W. Liu,; W.Gai, " The Undulator Based ILC Positron Source: Production and Capturing Simulation Study - Update", [ILC-NOTE-2007-009] http://ilcdoc.linearcollider.org/record/6947

[20] V.Bharadvaj, "May 2007 ILC Positron Source Update", a Talk at ILCWS2007, Hamburg, see ILCWS website- http://lcws07.desy.de/.

[21] A. Mikhailichenko, "The status of positron conversion System Development at Cornell", ILCWS2007, Proceedings, http://lcws07.desy.de/ , CBN 07-6, Cornell LEPP, see:

http://www.lns.cornell.edu/public/CBN/2007/CBN

07-6/CBN07-6.pdf

[22] W. Heitler. "The Quantum Theory of Radiation". Third edition, Oxford, 1954.

[23] A.D.Bukin, "Choice of optimal Positron Converter for Low Energy Beam (in Russian), Preprint INP 90--100,Novcsibirsk, 1990.

[24] A.Mikhailichenko," Liquid Metal Target for ILC", MOPLS108, Published in EPAC06 Proceedings, Edinburg, June 26-30, 2006.

[25] A.Mikhailichenko," Issues for the Rotating Target”, CBN-07-02, 2007. see:

http://www.Ins.cornell.edu/public/CBN/2007/CBN07-2/CBN07-2.pdf

[26] A.D.Bukin, A.A.Mikhailichenko, "Optimized target strategy for polarized electrons and positrons",

BUDKER-INP-1992-76, Oct 1992. 37pp., see: http://www-

project.slac.stanford.edu/lc/local/systems/Injector/Talks\%20and\%20Papers/PolPositronPapers/Buki nandMikhaili9276.pdf

[27] A. Mikhailichenko, "Polarized $e^{+}, e^{-}$Production Based on Conversion of Gammas, Obtained from Helical Undulator", CLNS 01/1758, Cornell University, Ithaca, NY 14850July 11, 2001, a talk at SNOWMASS2001

[28] F.Zhou, Y.Batygin, Y.Nosochkov, J.C.Sheppard, M.D.Woodley,"Start-to-end Beam Optics Development and Multi-particle Tracking for the ILC Undulator-Based Positron Source", slac-pub12239, Jan 2007. http://www.slac.stanford.edu/cgi-wrap/getdoc/slac-pub-12239.pdf

[29] Yu. Ivanyushenkov, et. al. "Development of a Full Scale Superconducting Undulator Module for the ILC Positron Source”, THPMN071, PAC2007. 